Meta

Journal des traducteurs

Translators' Journal

\title{
Changement sémantique et terminologie
}

\section{Silvia Pavel}

Volume 36, numéro 1, mars 1991

La terminologie dans le monde : orientations et recherches

URI : https://id.erudit.org/iderudit/003805ar

Aller au sommaire du numéro

Éditeur(s)

Les Presses de l'Université de Montréal

ISSN

0026-0452 (imprimé)

Découvrir la revue

Citer cet article

Pavel, S. (1991). Changement sémantique et terminologie. Meta, 36(1), 41-48.

Ce document est protégé par la loi sur le droit d'auteur. L'utilisation des services d'Érudit (y compris la reproduction) est assujettie à sa politique d'utilisation que vous pouvez consulter en ligne.

https://apropos.erudit.org/fr/usagers/politique-dutilisation/
Cet article est diffusé et préservé par Érudit.

Érudit est un consortium interuniversitaire sans but lucratif composé de l’Université de Montréal, l'Université Laval et l'Université du Québec à Montréal. Il a pour mission la promotion et la valorisation de la recherche. https://www.erudit.org/fr/ 


\section{CHANGEMENT SÉMANTIQUE ET TERMINOLOGIE}

Silvia Pavel

Secrétariat d'État du Canada, Montréal, Canada

Chaque science, chaque art, chaque métier, en composant sa terminologie marque de son empreinte les mots de la langue commune. (M. Bréal, Essai de sémantique, 1897)

D'Aristote à Fontanier, la polyvalence des mots fut expliquée en partie par les limites de la mémoire, par l'indésirabilité de l'univocité mot-sens, par l'inadéquation inévitable entre le monde des mots et celui des idées. «La multitude et la variété infinie de nos perceptions et de nos idées augmente toujours par le progrès même des langues. Quelle mémoire suffirait à apprendre tant de mots et à les retenir, à les reproduire?... quelle mémoire n'en serait pas accablée, étouffée? Tandis que dans l'ordre actuel des choses, la mémoire la plus commune peut sans peine, avec un nombre de mots assez borné, fournir de quoi exprimer un nombre infini d'idées.» (Fontanier 1834: 158).

La polysémie est un phénomène universel des langues, mais son bien-fondé dans la langue scientifique fut longtemps contesté, par certains théoriciens de la terminologie, au profit de l'idéal de monosémie. Pourtant, les termes créés par néologie de sens se comptent en dizaines de milliers et des concepts nouveaux surgissent sans cesse aux points de convergence des disciplines établies, que ce soit par la récupération partielle des contenus significatifs préexistants, par la migration et l'appropriation interdisciplinaire de concepts en place, ou encore par leur substitution pure et simple:

«As John Locke put it, it is impossible that every particular thing should have a distinct particular name. Words, therefore, and linguistic representations in general lose information every time they reduce knowledge or experience to language... Aristotle thought, and his shadow has stretched further down the millenia than most other ancient philosophers, that essences or fixed species of things, each of which could be given its correct name or definition, were the goal of science, and that reality was cut up naturally into such natural essences or species, related by universal causal laws which could be discovered. Now there is no sufficient reason to suppose that this metaphysics of nature is correct.» (M. Hesse 1989)

Les modifications de sens (acception) des mots - l'extension, la restriction, la suppression ou la substitution - furent étudiées par la rhétorique classique en tant qu'opérations fondamentales de la pensée et définies par rapport à la dichotomie sens propre/sens figuré - le sens propre ne correspondant pas nécessairement au sens étymologique ou primitif, mais au sens perçu comme usuel, habituel, ordinaire d'un mot, et le sens figuré ou tropologique étant considéré comme prêté à un mot soit par le besoin de combler une lacune de désignation, soit par le désir d'obtenir un effet esthétique inédit. Ainsi Fontanier distingue-t-il cinq espèces de sens métaphoriques obtenus par le transfert de caractères propres d'une chose à une autre: animé-animé (ruminer dans la tête), inanimé physique-inanimé moral (le vaisseau de l'État), inanimé-animé (tout 
homme est un abîme), animé physique-inanimé physique (l'incendie dévore tout), animé moral-inanimé moral (l'expérience est la maîtresse des arts).

Les tropes d'usage - créés par nécessité, ou forcés - appelés catachrèses et produisant «des sens propres de seconde origine, intermédiaires entre le sens propre et le sens figuré», furent classés (Fontanier: 213-219) en catachrèse de métonymie de la cause (ex. un Rubens - pour un tableau de ce peintre), de la chose (un masque - pour une personne masquée), du contenant (les tribunes - pour les auditeurs des tribunes), du contenu (la Comédie - pour le lieu où l'on la joue); catachrèse de synecdoque de la partie (bouches - personnes à nourrir), de la matière (argent - pour la monnaie en argent), d'abstraction (le gouvernement - pour les gouvernants), du genre pour l'espèce, de l'espèce pour l'individu et de l'individu pour l'espèce (un Tartuffe - un hypocrite dangereux); et catachrèse de métaphore qui «embrasse toutes les espèces de mots représentatifs d'idées... jusqu'à l'interjection». Mais malgré leur expressivité, et leur étonnante productivité dans les langues spéciales (on admettait volontiers qu'il s'en produisait plus en un jour de halle qu'en plusieurs séances d'Académie), ces figures d'usage, leur nature, leurs causes génératrices et leur rôle dans l'évolution du langage, prirent peu de place dans les traités de rhétorique.

En revanche, les tropes libres de l'écrivain - ou tropes-figures firent l'objet de nomenclatures et de définitions détaillées, sévèrement critiquées d'ailleurs par la stylistique moderne de Bally à Bruneau et à Spitzer: «la rhétorique ancienne, science réputée mercenaire et prescriptive [...] a réduit son domaine à une nomenclature de bizarreries codifiées: au mieux une taxinomie, pseudo-science ou archéo-science qui croit avoir compris la genèse des mécanismes de la pensée et de son expression pour avoir attribué un nom aux traits de langage les plus piquants. On peut estimer en effet que dans tous les domaines la science moderne est née au moment où les savants ont cherché à tracer les causes génétiques et dynamiques des phénomènes et de leurs enchaînements, au lieu de s'acharner à compléter des inventaires dont les articles, isolément les uns des autres, nommaient, classaient, décrivaient leurs objets dans une essence immuable, comme des archétypes chargés par la nature de perpétuer leur espèce ne varietur.» (Suhamy 1981: 12).

Au début du XIX ${ }^{\mathrm{e}}$ siècle, la rhétorique gréco-française et la philologie historique comparée de tradition allemande contribuèrent à l'apparition d'une nouvelle science des significations appelée «sémasiologie» par H. Reisig (1839), et «sémantique» depuis Michel Bréal (1897). La sémantique grammaticale de Reisig et de Darmestetter étudiait la vie des mots isolés, non pas celle du langage et ce, à l'aide de concepts empruntés soit aux sciences-mères (p. ex. l'extension et la compréhension des mots et les relations de contiguité, d'inclusion, de ressemblance dans la classification logico-rhétorique des changements sémantiques), soit à la biologie dont les théories évolutionnistes en firent la science «par excellence» du siècle.

Avec Stöcklein (1898), les mutations sémantiques reçoivent une interprétation psychologique et génétique. La signification du mot (rapport entre sa forme et son contenu significatif) est saisie dans le contexte phrastique, ses variations sont reliées à la situation de l'énonciation, placées dans l'espace mental des interlocuteurs, et expliquées par l'écart entre la représentation mentale du mot chez le locuteur et celle que lui associe l'auditeur. Il n'est plus question de significations fixes, hors contexte, mais plutôt de significations floues, s'y ajustant et se transformant suite aux insertions dans des contextes nouveaux. Des significations occasionnelles se substituent aux significations usuclles des mots par répétition de l'association mot-nouvelle représentation et par participation à la création de nouvelles combinaisons. 
D'autre part, tout comme Bréal qui voyait dans la disproportion entre mot et chose une source permanente de restrictions et d'extensions de sens, Stöcklein perçoit dans l'inadéquation de l'expression à la chose, renforcée par le changement constant des objets qui nous entourent, une forme de changement sémantique qu'il appelle ajustement et qu'il place dans l'espace référentiel des interlocuteurs. Cet ajustement se constate tout aussi clairement et fréquemment à la lecture de tout rapport de recherche, de communication scientifique ou de monographie spécialisée qui contient une critique des idées avancées ailleurs, ou une contribution originale de l'auteur, où il peut prendre la forme d'une stipulation telle:

«The notion of frame used in this essay is the result of a mixture of AI jargon, the German term Feld, the French term champ (sémantique) and Goffman's frame-space (Nerlich et Clarke 1988: 83).

L'interprétation psycho-génétique fut développée par L. Roudet (1921) et H. Sperber (1923) qui essayent de comprendre pourquoi une signification nouvelle remplace une autre, qu'est-ce qui détermine sa production, son acceptation, sa diffusion. Ils trouvent la source du renouvellement dans la charge affective de l'emploi d'un mot, dans la force expressive qui le propulse d'un langage particulier dans le langage courant. Sperber illustre cette irruption des significations à haute charge affective d'un domaine de représentations restreint dans d'autres sphères d'emploi, par la prolifération des métaphores militaires dans la vie familiale et par la multiplication ultérieure des synonymes familiers pour le mot mitrailleuse. Mais il ne met pas ce phénomène linguistique en rapport avec les conditions de la connaissance scientifique étudiées par G. Bachelard (1934). Rien n'est dit non plus sur l'irruption des terminologies d'une science dans une autre, d'une science dans une technique ou d'une science dans les arts. En résumant la théorie de Sperber, Nerlich (1988) cite des termes de l'informatique tels interface et user-friendly employés en langage courant avec le sens échange et facile à faire. Un exemple similaire est, en français, l'emploi du terme intégré en agriculture et en économie (agriculture intégrée, développement intégré pour angl. sustainable development) par analogie à production, fabrication intégrée par ordinateur, ou la métaphorisation de termes appartenant à la géométrie fractale lors de l'apparition récente de l'art fractal assisté par ordinateur. L'on pourrait sans doute mettre en rapport cette notion de charge affective et celle, plus récente, de pertinence développée par Dan Sperber et D. Wilson (1989).

A. Meillet (1905) déplace l'investigation du changement sémantique du mot vers le langage en soulignant sa dimension sociale. Il cherche à identifier «les actions qui déterminent les innovations et les conservations dont l'ensemble constitue l'histoire du langage» et voit dans le caractère essentiellement discontinu de la transmission du langage «la condition première qui détermine la possibilité et les modalités de tous les changements linguistiques». Aussi l'enfant qui apprend à parler ne reçoit-il pas la langue toute faite mais la recrée tout entière à son usage d'après ce qu'il entend autour de lui. Sans contester l'analyse psycho-génétique selon laquelle tout changement sémantique ne peut avoir lieu sans être provoqué par une action définie, Meillet soutient néanmoins que celle-ci n'en fournit pas une explication suffisante des causes efficientes. Il distingue les causes proprement linguistiques du changement déterminé par la structure de la phrase, et les causes extra-linguistiques du changement entraîné par la variation des choses et des notions auxquelles sont associés les mots. Mais il estime que le plus grand nombre des changements de sens procèdent d'un troisième ordre de causes à savoir, «la répartition des hommes de même langue en groupes distincts», professionnels ou autres. Le principe essentiel du changement sémantique réside donc dans un fait de structure sociale. 
«Les faits linguistiques, les faits historiques et les faits sociaux s'unissent, agissent et réagissent pour transformer le sens des mots: le moment essentiel est le passage d'un mot de la langue générale à une langue particulière, ou le fait inverse, ou tous les deux. Les changements de sens doivent être considérés comme ayant pour cause principale la différenciation des éléments qui constituent les sociétés.» (p.38)

Selon Meillet, les mots de la langue commune acquièrent un sens restreint ou spécialisé en passant dans l'usage d'un groupe particulier, et inversement, ils élargissent leur sens, deviennent plus généraux lorsqu'ils sont adoptés par un cercle plus étendu, de sorte que la généralité d'un sens est souvent proportionnelle à l'étendue du groupe qui l'emploie. Par exemple, viande signifiait aliment dont se nourrit l'homme avant de recevoir le sens restreint chair animale dont se nourrit l'homme, tandis que panier signifiait corbeille à pain avant de prendre le sens plus général réceptacle servant à contenir, à transporter des marchandises, des provisions, des animaux. Le changement de sens peut ajouter au mot une valeur négative (dans le vocabulaire de la fauconnerie niais signifiait qui n'est pas encore sorti $d u$ nid avant de qualifier en langue courante quelqu'un dont la simplicité, l'inexpérience va jusqu'à la bêtise) ou au contraire, une valeur positive (maréchal signifiait garçon d'écurie avant de prendre le sens haut fonctionnaire $d u$ roi $)$. L'amélioration et la péjoration prennent souvent les formes classiques de l'euphémisme, de l'hyperbole et de la litote (p. ex. en informatique, étreinte fatale et bombe logique, ou en anglais de I'IA le nouveau sens mélioratif to hack travailler passionnément, employé par opposition au néologisme péjoratif to tool travailler avec acharnement mais sans inspiration ni plaisir).

De plus, les groupes professionnels «ont à désigner un grand nombre d'objets et de notions pour lesquels la langue commune n'a pas de noms parce que le commun des hommes ne s'en occupe pas». Beaucoup de ces désignations sont obtenues en attribuant à des objets le nom d'autres objets avec lesquels ceux-ci ont une ressemblance plus ou moins lointaine (catachrèse de métaphore), y sont normalement associés (catachrèse de métonymie) ou y sont normalement inclus (catachrèse de synecdoque). Ces «noms d'autres objets» provenant soit de la langue courante soit d'une autre langue de spécialité abondent en informatique : des métaphores iconiques désignent des symboles graphiques (angl. le symbole ! est appelé bang, shriek, ou wow, le symbole@ — whirlpool, le symbole \# — mesh, crunch, pig-pen); les métaphores botaniques employées en mathématiques (arbre, forêt, feuille, racine, branche, sommet) passent globalement en informatique où elles génèrent de nouveaux emplois métaphoriques (affectation sylvestre, élagage, arbre touffu); les métaphores anthropomorphiques prolifèrent en intelligence artificielle (p. ex. les opérations effectuées sur les graphes de raisonnement portent des noms tels recul, saut, escalade, descente, raccourci, qui caractérisent normalement les mouvements des animés).

À l'intérieur d'une communauté professionnelle, le vocabulaire varie par suite à la tendance à marquer l'indépendance et l'originalité de ses membres, ou celle de la communauté en question vis-à-vis la société générale qui tend à uniformiser la langue. L'on notera ainsi des différences plus ou moins marquées dans la définition de la notion démon selon l'usage qu'en font des chercheurs IA tels Selfridge, Minsky, Hewitt, Charniak ou McDermott, par rapport à l'usage répandu chez les informaticiens.

Parmi les facteurs facilitant l'innovation sémantique dans les groupes professionnels, Meillet cite la cohérence de l'univers référentiel, la stabilité des échanges locuteur-auditeur (mêmes associations d'idées, mêmes manières de s'exprimer, entendement à demi-mots) et le souci de demeurer inintelligible au profane. Ainsi, comme on pouvait s'y attendre, un des passe-temps favoris de l'intelligentsIA américaine est la lecture de bandes dessinées et de romans de science-fiction. Comme tous les 
intéressés sont au courant du vocabulaire véhiculé dans ces ouvrages, les transferts lexicaux vers le vocabulaire (ou plus précisément l'argot) de l'IA se trouvent grandement facilités. Par exemple, le verbe martien «to grok» inventé par Robert Heinlein dans son roman Stranger in a Strange Land avec le sens métaphysique «être un avec» passe en IA avec le sens «comprendre vraiment toutes les conséquences d'un changement de programme», tandis que gronk - dernier soupir d'un sympathique dinosaure dans la bande dessinée B.C. de Johnny Hart - prend en IA le sens verbal «cesser de fonctionner» (angl. to gronk out). L'intérêt de ces exemples réside tant dans l'origine artistique ou littéraire de l'innovation terminologique que dans le greffage de néosémantismes technoscientifiques sur des néologismes de forme.

D'autres facteurs innovateurs sont l'hétérogénéité sociale, géographique et linguistique des communautés professionnelles qui favorise le changement sémantique par fusion dialectale et sociolectale, et la réceptivité aux influences étrangères qui explique les emprunts sémantiques et les traductions littérales de termes étrangers. A ce chapitre, Meillet souligne les importantes ressemblances interlinguistiques des vocabulaires techniques et scientifiques: «le vocabulaire militaire est à peu près le même d'un bout de l'Europe à l'autre; les savants créent des vocabulaires spéciaux qui se répandent rapidement et comme la science est éminemment internationale, les termes particuliers inventés par les savants sont ou reproduits ou traduits dans des groupes qui parlent les langues communes les plus diverses. Ainsi, l'Europe doit à la scolastique la plus grande partie de ce que, dans la bigarrure de ses langues, elle a d'unité de vocabulaire et d'unité de sens des mots» (p.23). Aujourd'hui l'observation de Meillet s'applique non seulement à l'Europe mais au «village global» de McLuhan où le rôle de la scolastique est joué par les technosciences (y compris le vocabulaire militaire et savant), et celui du latin par l'anglais.

Quant aux facteurs facilitant la diffusion des innovations sémantiques à l'extérieur des groupements particuliers, Meillet mentionne la force des habitudes langagières d'un groupe dans ses échanges avec des individus qui lui sont étrangers, l'effet de mimétisme que le vocabulaire d'un groupe prestigieux provoque chez les individus qui n'y ont pas accès, et l'intégration des objets et des concepts spécialisés dans la vie courante soit par mode soit par nécessité. La littérature de vulgarisation, la télévision éducative et les communications par satellite mettent ces innovations à la portée de tous.

Meillet est un des premiers à avoir saisi le caractère universel de l'emprunt figuré aux langues spéciales («Les faits de ce type sont si naturels qu'on les voit se reproduire de façon indépendante dans des langues diverses et à des époques diverses» p. 28), mais il l'assimile à la généralisation motivée par l'expressivité et n'y voit pas un processus indépendant de changement de sens. D'autre part, il note la complexité des emprunts internes, les séries successives d'emprunts et d'emplois figurés par lesquels le sens des mots se transforme (de la langue courante à la langue spéciale, de la langue spéciale à la langue courante et même d'une langue spéciale à une autre), leurs effets divers sur la langue des groupes sociaux qui les adoptent simultanément (c'est ce que Kocourek appelle de nos jours navettes d'emprunt) et signale une certaine polarisation des types de changements sémantiques par couches sociales distinctes. Toute son argumentation se fonde sur le caractère universel mais secondaire des aspects psychiques du changement en opposition avec la pertinence de ses aspects sociaux:

«Les conditions psychiques de la mutation sémantique sont constantes; elles sont les mêmes dans les diverses langues et aux diverses périodes d'une même langue; si donc on veut expliquer la variation, il faut introduire la considération d'un élément variable lui-même, et, étant donné les conditions du langage, cet élément ne peut être que la structure de la société où est parlée la langue considérée.» (p.33) 
Avec Gustaf Stern (1931) et Steven Ullman (1952), la sémantique devient une discipline linguistique autonome au même titre que la phonologie ou la syntaxe. On cherche à dépasser l'idiosyncrasie et l'imprévu, à trouver des régularités, des lois du changement sémantique et à en expliquer les causes et la nature. Stern définit le changement externe comme résultant du renouvellement du référent et le divise en substitutions objectives (ex. l'évolution sémantique du mot plume suit les perfectionnements de l'instrument à écrire), cognitives (p. ex. le sens de atome a changé avec nos connaissances sur la notion) et subjectives (p. ex. l'amélioration et la péjoration reflètent souvent des changements d'attitude affective vis-à-vis du référent). Malgré l'importance qu'elles ont acquise en lexicographie française (Matoré, Quemada), ces distinctions fondamentales pour l'analyse conceptuelle restent peu connues en terminologie où l'on se limite encore aujourd'hui à une structuration aristotélicienne, statique des concepts soit sur l'axe vertical des relations générique - spécifique, soit sur l'axe horizontal des co-hyponymes sans se préoccuper de leur devenir.

Ullmann classe les modifications par renouvellement du référent dans la catégorie des changements dus au conservatisme linguistique et les distingue des changements par innovation linguistique où le réseau associatif d'un mot ou d'une idée devient tellement important qu'il entraîne l'apparition de nouveaux sens dérivés soit par transfert de noms entre notions similaires (métaphores) ou contiguës (métonymie), soit par transfert de sens entre mots similaires (étymologie populaire) ou contigus (ellipse par conversion de catégorie grammaticale). Son interprétation des mécanismes du changement sémantique est critiquée et complétée par D. Geeraerts (1983) dans le cadre plus général de la théorie des prototypes conceptuels.

Henning Andersen (1973) réalise une nouvelle synthèse: il fusionne les classifications de Stern et de Ullmann dans une typologie structuraliste du changement linguistique en général, basée sur la théorie des traits distinctifs et les fonctions de Jakobson (référentielle, connative, émotive, poétique, métalinguistique, phatique) qui montre la symétrie des régularités aux niveaux phonétique et sémantique. Il divise le changement sémantique en changement grammatical et lexical, différencie les innovations évolutives (basées sur la structure linguistique) des innovations adaptatives (basées sur le système communicatif), relie le changement sémantique externe de Stern à la fonction référentielle du langage et y applique la distinction introduite par Coseriu (1958) entre innovation et changement: «Innovations lead to change only if they are adopted, or generalized, by the members of a speech community. Innovations are made by individuals, but change is a social fact.» A. Rey (1976) pousse l'étude du sémantisme lexical au-delà du paradigme structuraliste et oriente la lexicologie française vers un nouveau bassin d'attraction des sciences du langage - la pragmatique — ou étude des signes en situation d'emploi. Sa contribution à l'élaboration d'une méthodologie de recherche terminologique et à l'étude du néologisme terminologique n'a rien perdu de son actualité.

Louis Guilbert (1975) étudie la créativité lexicale d'un point de vue générativiste. Il tente de saisir la dynamique (variation/ régulation) des mutations sémantiques et les implications socio-linguistiques du néologisme de sens en examinant les rapports entre la langue générale et les terminologies spécialisées, entre le niveau du discours et le niveau de la langue, entre la structure sémantique du langage et le cadre syntaxique du néosémantisme :

«Nous pensons que chaque création humaine, sur le plan du référent, provoque une réorganisation de la structure sémantique du signifié. Le contenu de signification d'un terme s'établit dans un temps donné par la constitution d'un faisceau de sèmes qui le définissent. Chaque modification de référent entraîne une réorganisation des relations entre les sèmes, la 
disparition de certains, l'apparition de nouveaux, l'abstraction nécessaire pour saisir le lien sémantique entre les nouveaux emplois et les emplois antérieurs se construisant à mesure de l'évolution de l'expérience et de sa pénétration dans la conscience linguistique de la communauté.» (p.72)

Sous l'influence des sciences cognitives, l'étude du changement sémantique se concentre de plus en plus sur la découverte de lois universelles telles la progression du concret vers l'abstrait (loi de Kronasser), ou du moins abstrait vers le plus abstrait, et l'analyse des processus cognitifs sous-jacents. S'inspirant des travaux de Fillmore (1977), de Rosch (1978), de Lakoff (1977) et de Minski (1975), l'approche cognitive applique les concepts de la logique floue au changement lexical et grammatical, aux catachrèses aussi bien qu'aux «vraies figures», et relie l'analyse du sens à celle des concepts, de leur formation et de leur acquisition. Traugott (1985) démontre, par exemple, que la loi de Kronasser s'applique à tous les types de changement (généralisation et spécialisation, amélioration et péjoration, métaphore et métonymie) observés dans certains champs sémantiques:

«When we turn from domains [...] such as natural kinds like bears or institutional kinds like kings, to domains associated with conceptual structure and mental life, for example time, causation, understanding, and engaging in speech events, the picture that emerges is one of regularity, not idiosyncrasy. The best way to get at this regularity is to focus on shifts from one semantic domain to another» (p.159).

Ainsi, nous fait-on remarquer, les termes désignant l'ouie et la vue prennent dans plusieurs langues le sens intellectuel comprendre: fr. je vois, bien entendu, angl. insight, wit (dérivé du latin videre $=$ voir). Les termes désignant le toucher, l'odorat, le goût expriment des préférences et des valeurs personnelles (on ne peut pas sentir quelqu'un parce qu'il a mauvais goût, ou qu'il n'a pas un caractère doux). Les adjectifs d'intensité des couleurs, de la lumière sont associés à des habilités intellectuelles (un discours vibrant, un esprit brillant, une intelligence vive, une conversation terne, une performance éblouissante) tandis que les termes pour couleurs primaires qualifient des états affectifs (rouge de colère, vert d'envie, une peur bleue, rire jaune, avoir le foie blanc, être d'une humeur noire). Ainsi que Matore l'avait déjà montré, l'espace est une source inépuisable de verbes abstraits: les termes spatiaux sont employés avec un sens temporel (slow down, burn up); les verbes désignant des processus mentaux contiennent souvent un élément spatial : réfléchir, sous-entendre, inférer (lat. porter dans), déduire (lat. faire descendre), tout comme la plupart des verbes relatifs aux actes de parole: converser (lat. demeurer quelque part, avec quelqu'un), insulter (lat. sauter sur), prétendre (lat. tendre en avant), proférer (lat. porter en avant), répondre (lat. s'engager en retour), suggérer (lat. porter sous), supplier (lat. se plier à genoux). Autant que les métaphores poétiques, ces métaphores usées ou lexicales ont une fonction expérientielle. Lakoff and Johnson (1980) démontrent comment elles interviennent dans la formation et le développement des systèmes conceptuels, des cultures, de la pensée et de l'activité humaines.

L'approche cognitive éclaire de la sorte une caractéristique importante de la métaphorisation terminologique, à savoir la relation entre transparence motivationnelle et efficience conceptuelle:

«Given the general assumption that concepts are not clear-cut entities but vague collections of marginally deviant subconcepts, held together by their overall resemblance, it will be clear that methaphor fits in neatly: as Ullmann points out, the resemblance with the basic concept is the basis of metaphorical transfers. Word meanings and semantic categories can only be adequately described as dynamic wholes, loosely structured around prototypical senses. Semantic phenomena cannot be isolated from the way in which men experience the world 
and no adequate theory of language can be developed if it does not take into account the world knowledge of the language user, his experiences and so on» (Geeraerts: 232).

L'on y découvre un point de vue opposé à celui de l'approche aristotélicienne de l'analyse componentielle et de la sémantique logique selon laquelle le sens peut être défini à l'aide de catégories sémantiques nettement délimitées, et qui postule la différence entre signification et connaissance du monde, entre le contenu sémantique du mot, du terme et l'information encyclopédique sur leur référent. Quoique minoritaire en sémantique, ce point de vue nouveau ne manquera pas d'attirer l'attention des théoriciens de la terminologie qui s'intéressent à l'organisation des connaissances et à la systématisation conceptuelle dans les technosciences émergentes, au rapport entre le sens et le référent du néologisme terminologique, ou aux problèmes de stabilité et d'usage terminologiques dans les spécialités.

\section{BIBLIOGRAPHIE}

ANDERSEN, H. (1973): «Towards a Typology of Change: Bifurcating changes and Binary Relations», J.M. Anderson \& C. Jones (ed.), Historical Linguistics II, North-Holland, Amsterdam, pp. 17-61.

BACHELARD, G. (1934): Le nouvel esprit scientifique, Paris.

BRÉAL, M. (1897): Essai de sémantique, Paris, Hachette.

COSERIU, E. (1958) : Sincronia, diacronia e historia, Universidad de la Republica, Montevideo.

DARMESTETTER, A. (1887): La vie des mots étudiés dans leur signification, Paris.

FILLMORE, Ch. (1977): «Scenes-and-frames Semantics», A. Zampolli (ed), Linguistic Structures Processing, North Holland, Amsterdam.

FONTANIER, P. (1968): Les figures du discours (1830), réédité en 1968, Paris, Flammarion, avec une introduction par Gérard Genette.

GEERAERTS, D. (1983): «Reclassifying Semantic Change», International Journal of Theoretical and Applied Semantics, 4-2, pp. 217-240.

GROULT, M. (1989): «Histoire du vocabulaire scientifique en France», Le linguiste, 35.

GUILBERT, L. (1975): La créativité lexicale, Paris, Larousse.

HESSE, M. (1989): «Models, Metaphors and Myths», The New York Times, October 20, p. E-24.

KOCOUREK, R. (1982) : La langue française de la technique et de la science, Brandstetter Verlag, Wiesbaden.

LAKOFF, G. (1977): «Linguistic Gestalts», W.A. Beach et al. (eds), Papers from the l3th meeting of Chicago Linguistic Society, University of Chicago, Chicago.

LAKOFF, G, and M. JOHNSON (1980): Metaphors We Live By, University of Chicago, Chicago.

MEILLET, A. (1905-1906) : «Comment les mots changent de sens», L'Année sociologique, 9, pp. l-38.

MINSKI, M. (1975): «A Framework for Representing Knowledge», P.N. Winston \& Palmer, F.R. (ed), The Psychology of Computer Vision, McGraw-Hill.

NERLICH, B. (1988): «Théories du changement sémantique en Allemagne au XIX ${ }^{e}$ siècle», Histoire Epistémologie Langage, 10-1, pp. 101-112.

NERLICH, B. and D. D. Clarke (1988): «A Dynamic Model of Semantic Change», Journal of Literary Semantics, 17-2, pp. 73-90.

REISIG, H. (1839): Vorlesungen uber lateinische Sprachwissenschaft, Lehnhold, Leipzig.

REY, A. (1976): Le lexique : images et modèles, Paris, Armand Colin.

ROSCH, E.R. (1978): «Principles of Categorization», Rosch, E.R. \& Lloyd, B.B. (ed), Cognition and Categorization, Erlbaum, Hillsdale.

ROUDET, L. (1921): «Sur la classification psychologique des changements sémantiques», Journal de psychologie, 18, pp. 676-692.

SPERBER, H. (1923): Einfuhrung in die Bedeutungslehre, Scroeder, Leipzig.

SPERBER, D. et D. Wilson (1989) : La pertinence, Paris, Minuit.

STERN, G.(1931): Meaning and Change of Meaning, Bloomington, Indiana University Press.

STÖCKLEIN, J. (1898): Bedeutungswändel der Wörter, Lindaursche Buchhandlung, München.

SUHAMY, H. (1981): Les figures de style, coll. Que sais-je, Paris, PUF.

TRAUGOTT, E.C. (1985): «On Regularity in Semantic Change», Journal of Literary Semantics, 14-3, pp. 155173. 